\title{
Paroxysmal kinesigenic dyskinesia
}

\author{
AT Alibhoy ${ }^{1}$, Subhashie Wijemanne ${ }^{2}$ and Ranjanie Gamage ${ }^{3}$ \\ (Index words: Clinical features, response to carbamazepine)
}

\begin{abstract}
Paroxysmal kinesigenic dyskinesia (PKD) is a rare disorder characterised by brief and frequent attacks of abnormal involuntary movements induced by sudden movement. This disorder has not been reported previously in Sri Lanka. We studied six patients with respect to clinical presentation, aetiology, family history and response to treatment, and describe the Sri Lankan patterns of this illness. All the patients were males and the age at onset was from 11 to 22 years. The involuntary movements in all were dystonic and affected one or both sides, involving the face in the majority. All had difficulty in speaking during the attacks. One patient had an occasional attack during exercise. In all, the illness was sporadic, none had a family history of a similar illness and in none was it due to a secondary cause. The attacks usually lasted 10-60 seconds, and occurred up to 20 times a day. All patients responded well to anticonvulsants. PKD in Sri Lanka has a pattern similar to that described worldwide.
\end{abstract}

\section{Introduction}

Paroxysmal kinesigenic dyskinesia (PKD) is a rare condition characterised by brief and frequent attacks of involuntary movements induced by sudden movement. The symptoms are often misinterpreted by physicians and may pass unrecognised. We present six patients with PKD (Table 1).

\section{Case reports}

Six patients with PKD were evaluated over a period of 2 years. All patients were males, with the age of onset ranging from 11 to 22 years. In all, involuntary movements were dystonic, with twisting and abnormal posturing of the limbs. They usually involved both the upper and lower limbs. In three patients the attacks were unilateral, in one it was occasionally bilateral, and in two it was always bilateral. Most attacks were provoked by walking suddenly after a period of immobility. The limbs became dystonic making it impossible to continue walking, and the patients had to stay still until it resolved.

Case 1 had an occasional episode while running 25$100 \mathrm{~m}$, while Case 2 also developed attacks when frightened or startled. None had falls except Case 4 who had fallen on several occasions when he continued to walk. In four patients the mouth also deviated to a side. All of them could not speak during the event, and most would only smile during this period. None described a sensory aura. They usually had three to seven attacks daily, and had a maximum daily frequency of 20. Each episode lasted from 15 to 60 seconds.

None had a significant past history except Case 5 who had childhood generalised epilepsy treated with carbamazepine, which had been withdrawn after a 5-year seizure free period. Attacks of PKD appeared a year after stopping treatment. None gave a relevant family history. All patients responded well to carbamazepine. In Case 5, attacks reduced in frequency after the reintroduction of carbamazepine. Case 6 had been previously treated with phenytoin for a year, but presented again with recurrence of symptoms despite being on medication. He too responded well to carbamazepine.

\section{Discussion}

Paroxysmal dyskinesias are a group of disorders characterised by attacks of abnormal, intermittent,

Table1. Characteristics of the six patients with paroxysmal kinesigenic dyskinesia

\begin{tabular}{lcccccc}
\hline & Case 1 & Case 2 & Case 3 & Case 4 & Case 5 & Case 6 \\
\hline Age (years) & 15 & 13 & 12 & 19 & 24 & 20 \\
Symptoms (months) & 6 & 2 & 6 & 10 & 24 & 90 \\
Side-unilateral or bilateral & Uni & Uni & Bi & Bi & Uni & Usually Uni \\
Site & Limbs & Limbs and face & Limbs & Limbs and face & Limbs and face & Limbs and face \\
Falls & No & No & No & Yes & No & No \\
Max freq/d & 4 & 3 & 2 & 20 & 15 & 20 \\
Attack duration (seconds) & $15-60$ & $10-15$ & $30-60$ & $20-30$ & $30-60$ & $15-30$ \\
Attacks while on treatment & Nil & Nil & Nil & Nil & Few & Nil \\
\hline
\end{tabular}

${ }^{1}$ Resident Neurologist, ${ }^{2}$ Senior Registrar, ${ }^{3}$ Neurologist, National Hospital of Sri Lanka, Colombo, Sri Lanka. Correspondence: ATA, e-mail: <atalibhoy@yahoo.uk>. (Competing interests: none declared). Received 20 December 2005 and accepted 23 December 2005. 
hyperkinetic movements, which may consist of dystonia, choreo-athetosis, ballismus or a combination of these. They occur spontaneously or may be precipitated by sudden voluntary movement, exercise, sleep or stress [1]. Paroxysmal dyskinesias are classified into four categories according to the precipitating events [1], and the following forms are recognised: PKD induced by sudden movement, paroxysmal non-kinesigenic dyskinesia (PNKD) occurring spontaneously, paroxysmal exertion-induced dyskinesia (PED), induced after prolonged exercise, and paroxysmal hypnogenic dyskinesia (PHD) occurring during sleep.

Paroxymal kinesigenic dyskinesia is precipitated by sudden voluntary movement, especially after a period of rest. Less commonly, it occurs while talking, yawning or when startled [2]. Attacks that also occur during exercise, as seen in Case 1, have not been previously reported in PKD. Each attack usually lasts only a few seconds, but can last minutes to hours. They are usually unilateral, typically presenting as hemidystonia, but can be bilateral, and affect the limbs more often than face and trunk [3]. They may be greatly disabling, interfering with daily activities. Some have an inability to speak during the attacks. The attacks are often preceded by a "sensory aura", such as muscle tension, stiffness, and paraesthesia [3], which could not be elicited in any of our patients.

Paroxymal kinesigenic dyskinesia appears to be more common in males. The onset is usually in childhood or adolescence. Most cases are idiopathic, either sporadic or familial with an autosomal-dominant pattern of inheritance in most families. Some cases of symptomatic PKD have been reported, the most common being in multiple sclerosis. Other causes include stroke, head injury, metabolic abnormalities, and encephalitis [4,5].

The frequency of attacks tends to diminish with age and, in some, spontaneous remissions have been reported. PKD responds well to carbamazepine, despite lack of evidence of it being a seizure disorder [2]. In conclusion, PKD in Sri Lanka has a profile similar to that described worldwide.

\section{References}

1. Demirkiran M, Jankovic J. Paroxysmal dyskinesias: clinical features and a new classification. Annals of Neurology 1995; 38: 571-9.

2. Houser MK, Soland VL, Bhatia KP, Quinn NP, Marsden CD. Paroxysmal kinesigenic choreoathetosis: a report of 26 patients. Journal of Neurology 1999; 246: 120-6.

3. Bhatia KP. The paroxysmal dyskinesias. Journal of Neurology 1999: 246: 149-55.

4. Blakeley J, Jankovic J. Secondary causes of paroxysmal dyskinesias. Advances in Neurology 2002; 89: 401-20.

5. Bhatia KP. Secondary causes of paroxysmal dyskinesia. Journal of Neurology 1999; 246: 120-6.

\title{
Song
}

(Oliver Goldsmith, 1730 - 1774)

\author{
WHEN lovely woman stoops to folly, \\ And finds too late that men betray, \\ What charm can sooth her melancholy, \\ What art can wash her guilt away?
}

The only art her guilt to cover,

To hide her shame from every eye,

To give repentance to her lover,

And wring his bosom - is to die.

(To write a story having for its hero an old country clergyman (Vicar of Wakefield, by Oliver Goldsmith), which young and old shall read with eager interest, is no small achievement. And this, to his lasting fame, Oliver Goldsmith has done). Editor $C M J$ 